\title{
A sparse Kaczmarz solver and a linearized Bregman method for online compressed sensing
}

\author{
Dirk Lorenz Stephan Wenger Frank Schöpfer Marcus Magnor
}

April 1, 2014

\begin{abstract}
An algorithmic framework to compute sparse or minimal-TV solutions of linear systems is proposed. The framework includes both the Kaczmarz method and the linearized Bregman method as special cases and also several new methods such as a sparse Kaczmarz solver.

The algorithmic framework has a variety of applications and is especially useful for problems in which the linear measurements are slow and expensive to obtain. We present examples for online compressed sensing, TV tomographic reconstruction and radio interferometry.
\end{abstract}

Keywords: Sparse solutions, compressed sensing, Kaczmarz method, linearized Bregman method, radio interferometry

\section{Introduction}

Sparse solutions of linear systems play a vital role in several active fields and form the backbone of the theory known as compressed sensing [1, 2. A prominent approach to compute such sparse solutions in the underdetermined case is to compute solutions with minimal $\ell^{1}$-norm and this is known as Basis Pursuit 3 . There exists a large body of literature to compute solutions to this problem and we only refer to the recent review 4. In this work we propose a framework that addresses two fundamental problems: 1) The problem may be too large to fit into the computer's memory and hence, only parts of the whole problem can be processed at once. 2) The measurement process may be very slow, i.e. it takes considerable time to obtain a new row of the measurement matrix and the corresponding entry in the right hand side. Our algorithmic framework to compute sparse solutions uses (in the extreme case) only a single row of the measurement matrix in each step. The framework is flexible enough to include "block processing" of the matrix and also allows for generalization to sparse solutions in dictionaries (also known as $\ell^{1}$-analysis minimization [5, 6]) and total variation minimization [7. Our work can be seen as a combination of the Kaczmarz-approach [8] (also known as ART [9]) and the linearized Bregman method [10] and we follow the framework laid out in [11. Note that a different and heuristic sparse Kaczmarz solver have been proposed in 12 and a methodology for online compressed sensing based on homotopy was proposed in [13, 14. 


\section{The sparse Kaczmarz solver and the linearized Bregman method}

\subsection{The Kaczmarz solver}

We consider a matrix $A \in \mathbf{R}^{m \times n}$ with $n>m$ with rows $a_{k}^{T}(k=1, \ldots m)$, a vector $b \in \mathbf{R}^{m}$ and aim to find solutions to the underdetermined system of equations $A x=b$. A classical iterative method that only uses a single row $a_{k}^{T}$ in each step is Kaczmarz's method 88. It consists of iterative projections onto the hyperplanes $H_{k}=\left\{x: a_{k}^{T} x=b_{k}\right\}$, i.e. in iteration $k$ we choose an index $r(k)$ and perform $x^{k+1}=P_{H_{r(k)}}\left(x^{k}\right)=x^{k}-\frac{a_{r(k)}^{T} x^{k}-b_{r(k)}}{\left\|a_{k}\right\|^{2}} a_{r(k)}$. The control sequence could be simply cyclic $(r(k)=(k$ $\bmod m)+1$ ) but also randomized sequences are of use (cf. [15] where a convergence rate is proven when one samples the rows with probability equal to their squared norm). Convergence can be ensured, if the control sequence picks up any index infinitely often [16] and we call these control sequences admissible. The Kaczmarz method converges for any system $A x=b$ that has a solution and moreover, it converges towards the least squares solution. Interestingly, a very small change in the algorithm makes it converge to a sparse solution:

Theorem 2.1 (Convergence of the sparse Kaczmarz method). Assume that Ax=b has a solution, let $\lambda>0, r$ be an admissible control sequence and denote by $S_{\lambda}(x)=\max (|x|-\lambda, 0) \operatorname{sign}(x)$ the soft shrinkage function (applied component-wise to a vector). Then for $z^{0}=x^{0}=0$ the iteration

$$
\begin{aligned}
& z^{k+1}=z^{k}-\frac{a_{r(k)}^{T} x^{k}-b_{r(k)}}{\left\|a_{r(k)}\right\|^{2}} a_{r(k)} \\
& x^{k+1}=S_{\lambda}\left(z^{k+1}\right)
\end{aligned}
$$

converges to a solution of

$$
\min _{x} \lambda\|x\|_{1}+\frac{1}{2}\|x\|^{2}, \quad \text { s.t. } \quad A x=b .
$$

Proof. The theorem follows from the theory developed in [1] as the sparse Kaczmarz method is a special instance of the BPSFP (Bregman projections for split feasibility problems): Consider the problem as a feasibility problem "find $x \in \bigcap_{k=1}^{m} H_{k}$ " (with the hyperplanes $H_{k}$ defined above) and define $f(x)=$ $\lambda\|x\|_{1}+\frac{1}{2}\|x\|^{2}$. Then the sparse Kaczmarz method is a special instance of BPSFP with Bregman projections w.r.t. $f$ and inexact stepsizes according to [11, Theorem 2.8].

Remark 1. The sparse Kaczmarz method converges much faster if an exact stepsize, as described in [11 Section 2.5.2], is used. There one updates $z$ as $z^{k+1}=z^{k}-t_{k} a_{r(k)}$ with exact stepsize $t_{k}$ given as the solution of the problem

$$
t_{k}=\underset{t \in \mathbf{R}}{\operatorname{argmin}} f^{*}\left(z^{k}-t a_{r(k)}\right)+t b_{r(k)} .
$$

Here $f^{*}$ is the convex dual of $f$, i.e. $f^{*}(z)=\frac{1}{2}\left\|S_{\lambda}(z)\right\|^{2}$, in other words, $t_{k}$ is a solution of the piecewise linear equation

$$
a_{r(k)}^{T} S_{\lambda}\left(z^{k}-t a_{r(k)}\right)=b_{r(k)} .
$$

\subsection{From sparse block-Kaczmarz to the linearized Bregman method}

The flexibility of the BPSFP-framework allows to devise a blockwise method as follows: Split the matrix $A \in \mathbf{R}^{m \times n}$ into $L$ blocks $A_{l} \in \mathbf{R}^{m_{l} \times n}$ (with $m_{1}+\cdots+m_{L}=m$ ) and set the corresponding part of the right hand side $b \in \mathbf{R}^{m}$ to $b_{l} \in \mathbf{R}^{m_{l}}$. Then each equation $A_{l} x=b_{l}$ has an affine linear space $L_{l}$ of 
solutions and the solution space of $A x=b$ is $\bigcap_{l=1}^{L} L_{l}$. The basic block-Kaczmarz method (with control sequence $r$ ) then reads as

$$
x^{k+1}=x^{k}-A_{r(k)}^{\dagger}\left(A_{r(k)} x^{k}-b_{r(k)}\right)
$$

(i.e. the current iterate is orthogonally projected onto $L_{r(k)}$ by the pseudo-inverse of $\left.A_{r(k)}\right)$. A similar treatment would be possible for the sparse Kaczmarz method, but the corresponding Bregman projection onto $L_{r(k)}$ is not straight forward. A simpler method is based on the following idea: For each block compute a hyperplane that separates the current iterate from $L_{r(k)}$ and then calculate the Bregman projection onto this hyperplane. The iteration is

$$
\begin{aligned}
t_{k} & =\frac{\left\|A_{r(k)} x^{k}-b_{r(k)}\right\|^{2}}{\left\|A_{r(k)}^{T}\left(A_{r(k))} x^{k}-b_{r(k)}\right)\right\|^{2}} \\
z^{k+1} & =z^{k}-t_{k} A_{r(k)}^{T}\left(A_{r(k)} x^{k}-b_{r(k)}\right) \\
x^{k+1} & =S_{\lambda}\left(z^{k+1}\right)
\end{aligned}
$$

and converges to a solution of [2] (cf. [11, Corollary 2.9]).

Remark 2. The stepsize $t_{k}$ was called "dynamic" stepsize in [11. Another (usually worse) alternative is $t_{k}=\left\|A_{r(k)}\right\|^{-2}$ but also an exact stepsize similar to Remark 1 is possible (cf. [11, Algorithm 1]) and usually leads to faster convergence.

In the extreme case of just one block $(L=1)$, the resulting iteration reads as

$$
\begin{aligned}
z^{k+1} & =z^{k}-t_{k} A^{T}\left(A x^{k}-b_{r(k)}\right) \\
x^{k+1} & =S_{\lambda}\left(z^{k+1}\right)
\end{aligned}
$$

and is precisely the linearized Bregman method from [10] (up to the stepsize $t_{k}$ which is set constant $t=\|A\|^{-2}$ there).

\section{Online Compressed Sensing}

As an illustration of the potential of the proposed method, we take the following compressed sensing scenario: Assume that a sparse quantity $x \in \mathbf{R}^{n}$ can be measured by linear measurements, i.e. that one can generate numbers $b_{k}=a_{k}^{T} x$ for some vectors $a_{k} \in \mathbf{R}^{n}$, and for simplicity we assume that the vectors $a_{k}$ contain Gaussian randomly distributed entries. Moreover, assume that it is both costly and timeconsuming to take one measurement. In the classical compressed sensing scenario one would estimate the sparsity of the unknown solution (i.e. the number of nonzeros in $x$ ), then calculate the number $m$ of measurements that is needed to guarantee that the solution of $\min _{x}\|x\|_{1}, a_{k}^{T} x=b_{k}, k=1, \ldots, m$ is the exact sparse solution and solve the optimization problem (or with $\|x\|_{1}$ replaced by $\lambda\|x\|_{1}+\frac{1}{2}\|x\|^{2}$ for some large enough $\lambda$, cf. [17, 18]). In our new framework, we can start solving

$$
\min _{x} \lambda\|x\|_{1}+\frac{1}{2}\|x\|^{2} \quad \text { s.t. } \quad a_{k}^{T} x=b_{k}, k=1, \ldots, l
$$

as soon as the first $l$ measurements have been taken. There are at least two different possibilities to do so:

1. Increasing cycle sparse Kaczmarz: Perform sweeps of the sparse Kaczmarz method (1) with $r(k)=(k \bmod l)+1$. 
2. Increasing linearized Bregman method: Perform linearized Bregman iterations (4) with $A^{l}=$ $\left[a_{1}, \ldots a_{l}\right]^{T}$ and $b^{l}=\left[b_{1}, \ldots, b_{l}\right]^{T}$.

To illustrate the performance of both methods we generated a vector $x \in \mathbf{R}^{n}$ with $n=1500$ and 20 nonzero entries, initialized with a matrix $A$ consisting of a single row. Then we started the increasing cycle sparse Kaczmarz and the increasing linearized Bregman method, added a row to $A$ every 0.1 seconds and plotted the relative residual $\left\|A_{l} x^{k}-b_{l}\right\| /\left\|b_{l}\right\|$ and the relative reconstruction error $\left\|x^{k}-x^{\dagger}\right\| /\left\|x^{\dagger}\right\|$ against time in Figure 1. One clearly observes an interesting phenomenon: The residual decreases as expected and in the beginning, it shoots up to a large value as soon as a new line is added to the linear system; but from some point on this is not true anymore and the residual stays small and most interestingly: This happens precisely when the reconstruction error drops down drastically, indicating that the true sparse solution has been found. This effect can be exploited to stop doing measurements much earlier than indicated by a precomputed number $m$ by just observing the evolution of the residual.
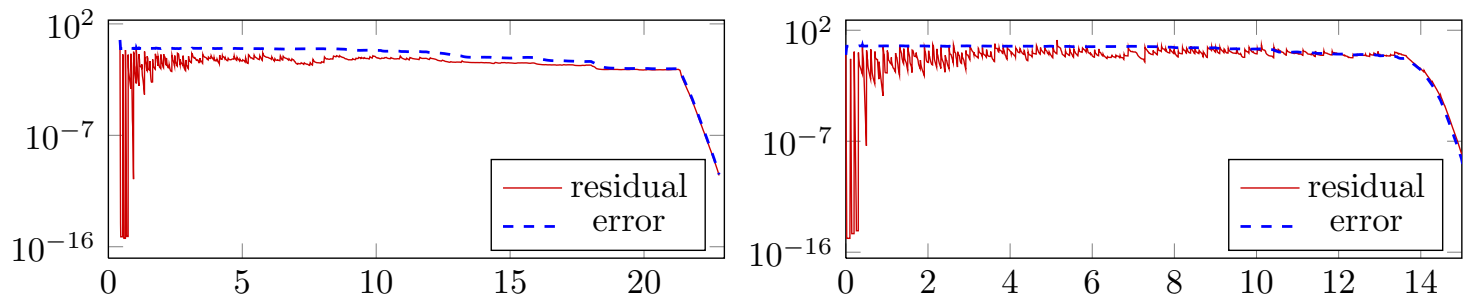

Figure 1: Illustration of the online compressed sensing scenario. Top: increasing cycle sparse Kaczmarz method against the computation time (final number of measurements is $m=196$ ). Bottom: increasing linearized Bregman method (final number of measurement is $m=150$ ).

\section{TV tomography}

To illustrate the flexibility of the BPSFP framework, we show a possibility to handle total variation minimization problems in a Kaczmarz style. The Kaczmarz method has proven to be an efficient tool in computerized tomography. It is used to compute least squares solutions in the case of an overdetermined system $A x=b$, i.e. minimizers of $\|A x-b\|^{2}$. To lower the dose of radiation it is desirable to reduce the number of X-ray projections. If the resulting linear system is underdetermined, further regularization is needed to obtain meaningful solutions. We consider a phantom of $128 \times 128$ pixels and 3128 measurements (i.e. approximately five times undersampling $)^{1}$, total variation regularization, i.e. we are interested in the solution of

$$
\min _{u}\||\nabla u|\|_{1} \quad \text { s.t } \quad A u=b
$$

(where $|\nabla u|$ stands for the Euclidean norm of the gradient of $u$, applied pointwise). As such the problem does not fit directly into our algorithmic framework and we propose to introduce an auxiliary variable $p$, add a quadratic regularization and reformulate (5) as

$$
\begin{aligned}
\min _{u, p} \lambda\||| p \mid\|_{1}+\frac{1}{2}\left(\|u\|^{2}+\|p\|^{2}\right) \quad \text { s.t } \quad & A u=b, \\
\nabla u & =p .
\end{aligned}
$$

\footnotetext{
${ }^{1}$ We use the AIRtools toolbox [19] to obtain the tomography matrix.
} 
Now we treat both constraints separately: The constraints $A u=b$ are treated as single hyperplane constraints $a_{k}^{T} u=b_{k}$. This leads to well known Kaczmarz steps for the $u$ variable: $u^{k+1}=u^{k}-$ $\frac{a_{r(k)}^{T} u^{k}-b_{r(k)}}{\left\|a_{r(k)}\right\|^{2}} a_{r(k)}$. For the constraint $B[u, p]^{T}=\nabla u-p=0$ we perform linearized Bregman steps (as the application of the full operator $B=[\nabla-I]$ is very cheap). Since the functional $f(p)=\lambda\|\| p \mid\left\|_{1}+\frac{1}{2}\right\| p \|^{2}$ does not allow for a simple calculation of exact stepsizes (cf. Remark 2), we use the dynamic stepsize as in (3) and get the linearized Bregman steps

$$
\begin{aligned}
w^{k} & =B[u, p]^{T}=\nabla u^{k}-p^{k}, \quad t_{k}=\frac{\left\|w^{k}\right\|^{2}}{\left\|B^{T} w^{k}\right\|^{2}} \\
{\left[\begin{array}{l}
v^{k+1} \\
q^{k+1}
\end{array}\right] } & =\left[\begin{array}{l}
v^{k} \\
q^{k}
\end{array}\right]-t_{k} B^{T} w^{k}, \\
u^{k+1} & =v^{k+1} \\
p^{k+1} & =S_{\lambda}^{2}\left(q^{k+1}\right)
\end{aligned}
$$

with the two-dimensional shrinkage function $S_{\lambda}^{2}: \mathbf{R}^{2} \rightarrow \mathbf{R}^{2}, S_{\lambda}^{2}(x)=\max (|x|-\lambda, 0) \frac{x}{|x|}$. The proposed framework allows to perform steps for either constraint in an arbitrary order. We show the results for the TV-Kaczmarz method where we perform either one linearized Bregman (LB) step per Kaczmarz sweep or $100 \mathrm{LB}$ steps per sweep, cf. Figures 2 and 3 .
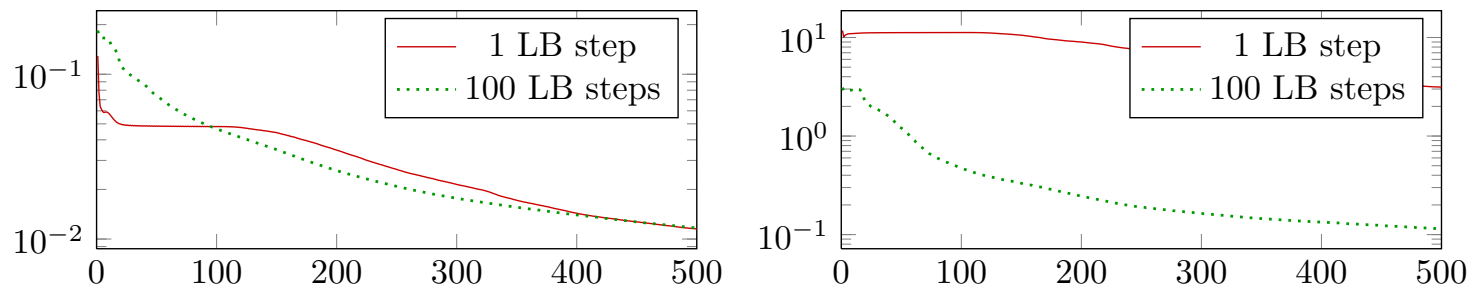

Figure 2: Illustration for the TV-Kaczmarz solver. Top: Relative resiudal $\left\|A u^{k}-b\right\| /\|b\|$, bottom: Residual $\|\nabla u-p\|$. Red solid: One linearized Bregman step per Kaczmarz sweep, green dotted: 100 linearized Bregman steps per Kaczmarz sweep.

\section{Radio interferometry}

In radio interferometry, multiple radio antennas record time-resolved amplitudes of radio emission from a small region of the sky [20. The correlation between the amplitudes at any pair of antennas defines a sampling point in the spatial Fourier representation of the image; its position is determined by the vectorial distance between the antennas. This sparse frequency sampling lends itself to reconstruction approches based on compressed sensing [1, 21, 22, 23, 24, 25].

A single, "snapshot" measurement results in a characteristic Fourier domain sampling pattern, 4 a. Since this pattern is very sparse, it often contains too little information for accurate reconstruction. However, over the course of a day, the sampling pattern changes with the rotation of the Earth. Thus, by combining the data from several measurements, much better coverage can be obtained, $4 \mathrm{~b}$.

Since observation time is scarce and expensive, it is desirable to only collect the minimum number of measurements necessary for reconstruction. Using an online reconstruction algorithm, 3 it is possible to 


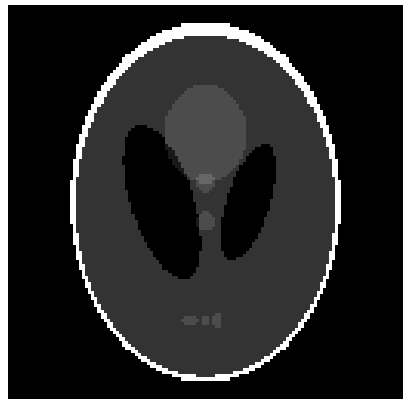

(a) Phantom.

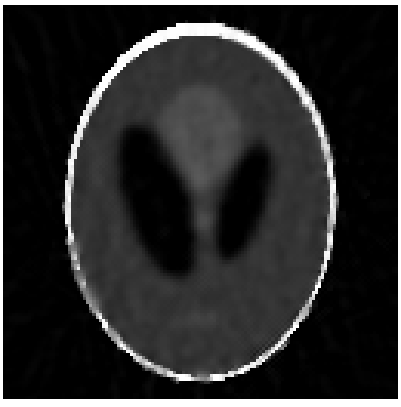

(b) 1 LB step per sweep.

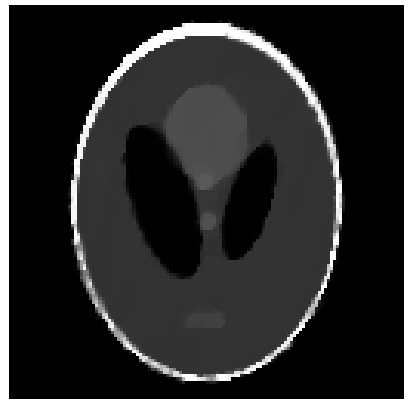

(c) 100 LB steps per sweep.

Figure 3: TV reconstructions with the TV-Kaczmarz solver.

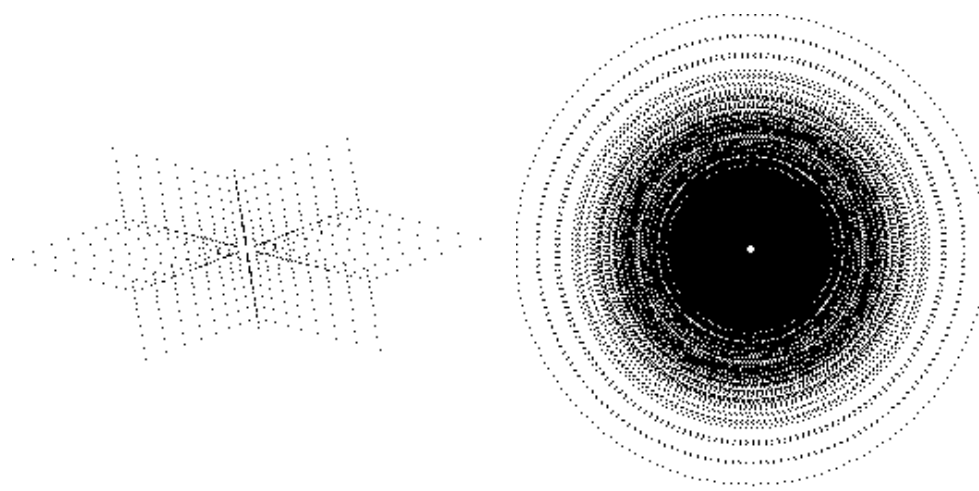

(a) Single snapshot.

(b) Half-day observation.

Figure 4: Interferometric sampling patterns.

continuously monitor the reconstruction result while new data is added. More importantly, the residual $\left\|A x^{k}-b\right\|$ provides feedback about the amount of information in each incoming data block: a new block that contains additional information instantly increases the residual because of the additional rows in $A$, while a redundant block causes no significant change in the residual. The absence of this increase in the residual value can serve as a stopping criterion for the measurement process.

We illustrate this application using simulated measurements of the radio source Sagittarius A West, 5a, in 7.5-minute intervals with the Very Large Array telescope. The sampling pattern of each 7.5-minute observational data block resembles a rotated copy of 4 a after a 12-hour observation, this results in the pattern shown in $4 \mathrm{~b}$, Each data block $b_{l}$ is related to the true image by a corresponding sampled Fourier transform $A_{l}$. While waiting for the next block, 300 iterations of (3) are performed on the blocks accumulated so far. Whenever a new data block is added, $r(k)$ starts with the newest block, followed by all others in a cyclic scheme. $\lambda$ is set to $10^{-4}\|x\|_{1}$, where $x$ is the ground truth image. Since negative intensities are not physically possible, we enforce the additional constraint $x \geq 0$ by truncating negative components of $z^{k+1}$ to zero before evaluating $S_{\lambda}\left(z^{k+1}\right.$ ) (a step that amounts to a Bregman projection 


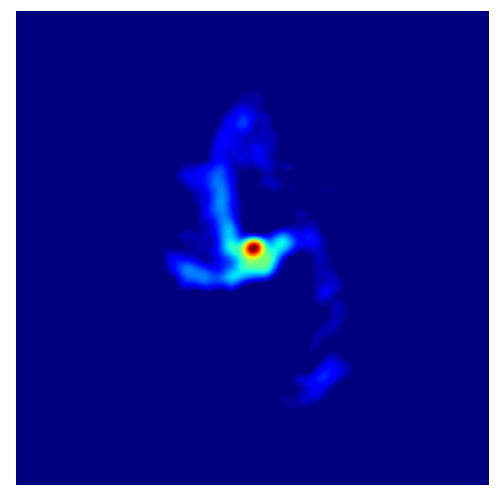

(a) Sagittarius A West.

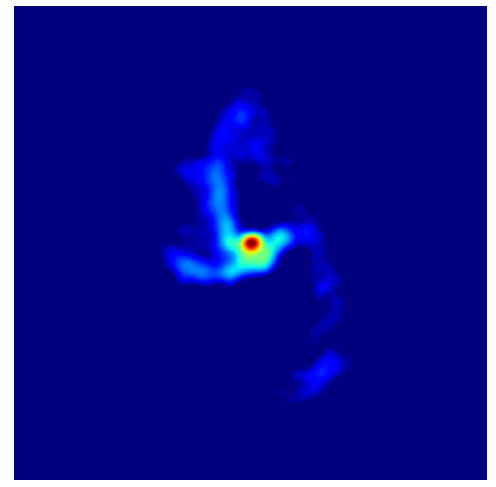

(b) Reconstruction.

Figure 5: Radio source and reconstruction from simulated data.

onto the non-negative orthant, cf. [11, Lemma 2.5]).

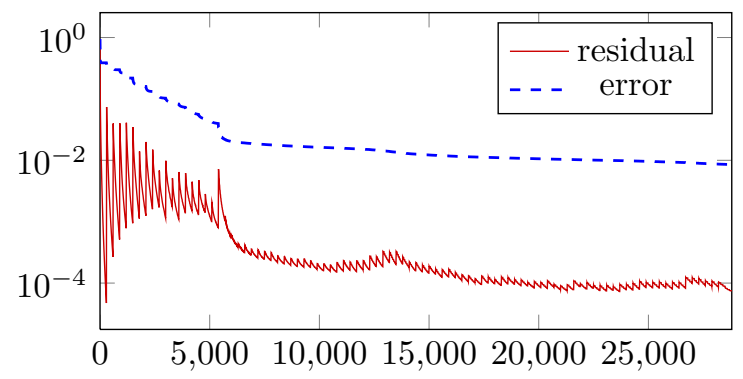

Figure 6: Relative residuals $\left\|A x^{k}-b\right\| /\|b\|$ and errors of online interferometric reconstruction after each time step $k$.

The residuals after each step are plotted in Figure 6. After 5400 iterations, when 18 blocks have been processed, additional data blocks stop causing a significant increase in the residual, and the relative reconstruction error abruptly drops from about $4 \%$ to about $2 \%$. In a practical setting, the measurement could now be stopped, based on an observation that relies only on the residual, freeing the telescope for other observations, and the algorithm could perform additional iterations on the available data until convergence. In our experiment, we successively add the remaining blocks, and the algorithm converges to a final relative error of about $0.85 \%, 5 \mathrm{~b}$.

\section{References}

[1] E. J. Candès, "Compressive sampling," in Proceedings of the International Congress of Mathematicians, 2006, vol. 3, pp. 1433-1452. 
[2] S. Foucart and H. Rauhut, A mathematical introduction to compressive sensing, Birkhäuser, Boston, 2013.

[3] S. S. Chen, D. L. Donoho, and M. A. Saunders, "Atomic decomposition by basis pursuit," SIAM Journal on Scientific Computing, vol. 20, no. 1, pp. 33-61, 1998.

[4] D. A. Lorenz, M. E. Pfetsch, and A. M. Tillmann, "Solving basis pursuit: Subgradient algorithm, heuristic optimality check, and solver comparison," Optimization Online E-Print ID 2011-07-3100, 2011.

[5] S. Vaiter, G. Peyre, C. Dossal, and J. Fadili, "Robust sparse analysis regularization," IEEE Transactions on Information Theory, vol. 59, no. 4, pp. 2001-2016, 2013.

[6] M. Haltmeier, "Stable signal reconstruction via $\ell^{1}$-minimization in redundant, non-tight frames," IEEE Trans. Signal Process., vol. 61, no. 2, pp. 420-426, 2013.

[7] A. Chambolle and P.-L. Lions, "Image recovery via total variation minimization and related problems," Numerische Mathematik, vol. 76, pp. 167-188, 1997.

[8] S. Kaczmarz, "Angenäherte Aufösung von Systemen linearer Gleichungen," Bull. Internat. Acad. Polon. Sci. Lettres A, pp. 355-357, 1937.

[9] R. Gordon, R. Bender, and G. T. Herman, "Algebraic reconstruction techniques (ART) for threedimensional electron microscopy and X-ray photography," Journal of Theoretical Biology, vol. 29, no. 3, pp. 471-481, 1970.

[10] S. Osher, Y. Mao, B. Dong, and W. Yin, "Fast linearized Bregman iteration for compressive sensing and sparse denoising," Communications in Mathematical Sciences, vol. 8, no. 1, pp. 93-111, 2010.

[11] D. A. Lorenz, F. Schöpfer, and S. Wenger, "The linearized Bregman method via split feasibility problems: Analysis and generalizations," Submitted for publication, September 2013, http://arxiv.org/abs/1309.2094.

[12] H. Mansour and O. Yilmaz, "A fast randomized Kaczmarz algorithm for sparse solutions of consistent linear systems," http://arxiv.org/abs/1305.3803, 2013.

[13] P. Garrigues and L. E. Ghaoui, "An homotopy algorithm for the lasso with online observations," in Advances in neural information processing systems, 2008, pp. 489-496.

[14] M. Salman Asif and J. Romberg, "Dynamic updating for $\ell_{1}$ minimization," Selected Topics in Signal Processing, IEEE Journal of, vol. 4, no. 2, pp. 421-434, April 2010.

[15] T. Strohmer and R. Vershynin, "A randomized Kaczmarz algorithm with exponential convergence," Journal of Fourier Analysis and Applications, vol. 15, no. 2, pp. 262-278, 2009.

[16] H. H. Bauschke and J. M. Borwein, "Legendre functions and the method of random Bregman projections," J. Convex Anal., vol. 4, no. 1, pp. 27-67, 1997.

[17] W. Yin, "Analysis and generalizations of the linearized Bregman method," SIAM J. Imaging Sci., vol. 3, no. 4, pp. 856-877, 2010. 
[18] F. Schöpfer, "Exact regularization of polyhedral norms," SIAM Journal on Optimization, vol. 22, no. 4, pp. 1206-1223, 2012.

[19] P. C. Hansen and M. Saxild-Hansen, "AIR tools - A MATLAB package of algebraic iterative reconstruction methods," Journal of Computational and Applied Mathematics, vol. 236, pp. 2167$2178,2012$.

[20] R. A. Perley, F. R. Schwab, and A. H. Bridle, Eds., Synthesis Imaging in Radio Astronomy, vol. 6 of Conference Series, Astronomical Society of the Pacific, 1989.

[21] Y. Wiaux, L. Jacques, G. Puy, A. M. M. Scaife, and P. Vandergheynst, "Compressed sensing imaging techniques for radio interferometry," Mon. Not. R. Astron. Soc., vol. 395, pp. 1733-1742, 2009.

[22] Y. Wiaux, G. Puy, and P. Vandergheynst, "Compressed sensing reconstruction of a string signal from interferometric observations of the cosmic microwave background," Mon. Not. R. Astron. Soc., vol. 402, no. 4, pp. 2626-2636, 2010.

[23] S. Wenger, S. Darabi, P. Sen, K.-H. Glassmeier, and M. Magnor, "Compressed sensing for aperture synthesis imaging," in Proc. IEEE International Conference on Image Processing (ICIP) 2010, Sept. 2010, pp. 1381-1384.

[24] S. Wenger, M. Magnor, Y. Pihlström, S. Bhatnagar, and U. Rau, "SparseRI: A compressed sensing framework for aperture synthesis imaging in radio astronomy," Publ. Astron. Soc. Pac. (PASP), vol. 122, no. 897, pp. 1367-1374, Oct. 2010.

[25] S. Wenger, U. Rau, and M. Magnor, "A group sparsity imaging algorithm for transient radio sources," Astronomy and Computing, vol. 1, pp. 40-45, Feb. 2013.

Dirk Lorenz

d.Iorenz@tu-braunschweig.de, Institute for Analysis and Algebra, TU Braunschweig, 38092 Braunschweig, Germany

Stephan Wenger, Marcus Magnor

\{wenger, magnor\}@cg.cs.tu-bs.de, Institut für Computergraphik, TU Braunschweig, 38092 Braunschweig, Germany

Frank Schöpfer

frank.schoepfer@uni-oldenburg.de, Carl von Ossietzky Universität Oldenburg, 26111 Oldenburg, Germany 